\title{
Pour une trace de sang
}

\section{Pascal Caron}

How can we bring together the trace, poetry and dance? Still today, Mallarmés texts influence the way that we can react to this question. Pascal Caron suggests the following answer: poetry joins dance through a trace of blood.

La trace réelle de la poésie est le sang d'énigmatiques menstrues. Je veux méditer sur cette proposition par le biais d'une autre pratique. Avec l'aide de l'art du biais, tant vis-à-vis de la pensée que du corps: la danse.

((Elle tournoie en traçant de ses cuisses humides des passages et des retraits. Elle s'écarte d'elle-même, imperceptiblement dans sa ronde. Ce n'est d'ailleurs pas une ronde. Un treillis décadenassé, un corps en fuite que l'air porte à l'endroit qu'il sera juste après, qu'il n'est pas encore dans cet instant. Une chasse sans proie. Elle zigzague une battue où sa meute aux abois la seconde - une meute qu'elle contient et lâche avec elle. Seuls des yeux affolés voient la meute, fixent cette femme qui ne se laisse pas épingler au carton du fantasme. Elle crève le regard et s'accroche aux pupilles englouties dans l'immobilité de la frayeur. Les pupilles qui la voient. Et qui croient justement percevoir la brillance de l'instant où elle tournoie sans écart avec elle-même et s'aiguise en contour. Là, elle; là, l'air. La frange d'extérieur est un apercevoir aussi fugace qu'invisible. Les pupilles qui la voient creusent des ténèbres, sont pleines d'obscurité.))

L'homme est un accessoire scénique, ridiculement réduit à la fonction de porteur ou de sauteur à son époque. Mais Stéphane Mallarmé se défend d'écrire l'apologie noire de la femme à la manière de certains de ses contemporains. Monstrueuse, fatale, bivalente (dotée de deux faces, toujours simples), elle est danseuse en vertu d'une métamorphose spatiale : glorieuse et éthérée sur scène,-déchue, charnelle et disponible en coulisse. Un poète de la fin du XIXe siècle se contente, le plus souvent, $d$ 'imiter et de représenter la belle que magnifie et protège la tour sacrée de l'art. Mêlé à la foule sous la pression de la nécessité, il chante l'effroi de voir un corps (le sien) devenu monnayable. Fortune de Salomé, d'Hérodiade, d'une Phèdre revisitées qu'un temps passé et 
présent croit soumises au bouillonnement du sang dans le giron, qu'un temps présent et passé rend maîtresses de tous les sangs. Au premier chef, celui de l'homme.

((Au sol les coulées de son ventre, y lire ceci: « À la nue accablante tu/ Basse de basalte et de laves/ À même les échos esclaves/ Par une trompe sans vertu ". Pour cela rien ne voltige ou ne papillonne d'autre quand elle bouge que l'empreinte continue de son ondulation liquide. Et ce flot d'incarnat qu'on croirait des ruisseaux passagers coulant à proximité de leur tarissement, "Avarement aura noyé/ Le flanc enfant d'une sirène ", non: fécondent le sol et drapent la vue d'une éternelle et sanguine AURORE BOREALE.))

L'axiome édicté par Mallarmé dans le texte "Ballets " marque, encore aujourd'hui, notre compréhension de la danseuse. Il établit le paradigme à partir duquel nous pensons, avec circonspection, le corps secondairement identifié comme féminin en train de pratiquer une énigme secondairement appelée danse:

À savoir que la danseuse n'est pas une femme qui danse, pour ces motifs juxtaposés qu'elle $n^{\prime} e s t$ pas une femme, mais une métaphore résumant un des aspects élémentaires de notre forme, glaive, coupe, fleur, etc., et qu'elle ne danse pas, suggérant, par le prodige de raccourcis ou d'élans, avec une écriture corporelle ce qu'il faudrait des paragraphes en prose dialoguée autant que descriptive, pour exprimer, dans la rédaction: poème dégagé de tout appareil du scribe.

((Le souffle des bras dans l'air, des hanches aux craquements intérieurs tus pour l'ouïe qui l'entend tournoyer en silence, des jambes balayant l'horizon ici même: vide. Hors d'elle, et c'est la terre qui respire sur son passage. D'une respiration liquide et chaude comme l'haleine d'un poisson qui garderait le plancton, les protistes errants, les lames de fond et le rêve d'une inspiration. Lui écrit: «Quel sépulcral naufrage (tu/ Le sais, écume, mais y baves)/ Suprême une entre les épaves/ Abolit le mât dévêtu ". Mouvement d'avant les bulles, d'avant leur crevaison inutile, et preuve de présence. De son geste incomplet d'expulsion.

Et un soupir immense au sol éternisant sa ronde.))

L'abolition des identités répond ici à un état historique de l'art scénique et à une exigence poétique singulière. Ni cet état de fait ni cette poétique ne peuvent être plus longtemps regardés comme des invariants: il est possible que la vérité nous aveugle. Et qu'elle 
dissimule d'abord la fragilité de la fiction qui la supporte. Chez Mallarmé, cette fiction: unique existence spirituelle de l'authenticité du " séjour terrestre "; unique valeur de tout art, de tout phénomène, de toute expérience, dans le Langage pérennisé par le livre; unique trace de l'événement, fulgurant, dans la célébration de sa Disparition.

((Elle semblait tenir quelque chose entre ses doigts, contre les doigts de son corps. Qu'elle échappe, rattrape juste avant le fracas, fait bondir et jouer de caresses sans toucher et de coups qui touchent à tous les coups. Quelque chose réduit en poussière par des doigts de labour; quelque chose enrayé pour la semaison. Un seul temps pour l'éclosion et la récolte. Elle semblait dévorer le fruit mûr sans altérer l'écorce d'aucune morsure. Et de l'énergie retrouvée, fouiller de nouveaux champs qui, une seconde plus tôt, n'existaient pas, des doigts de son corps.

Mais rien dans les mains, RIEN à voir blotti et précaire entre ses doigts.))

Le symbole qu'est la femme qui danse renverse la direction de l'attentat. La femme qui danse perd ce qu'un dévoilement réduit au statut $\mathrm{d}^{\prime}$ attributs. Mallarmé affirme cette condition de la danseuse parce qu'il voit toute trace dans l'abolition. On lui disait qu'il fallait concevoir la trace, mentale, dans un système de causes et de conséquences. Il mit sa parole en marge, et la danseuse avec elle, dans le monde de l'idée, de la métaphore, de la fiction. L'esprit du spectateur, gracieux par réflexion, confirme le " poème dégagé de tout appareil du scribe ". $\mathrm{Au}$ fond, bien que Mallarmé affirmât la nécessité $\mathrm{d}^{\prime}$ un lieu réel pour la danse (avec le mime, dans " Le Genre ou des Modernes »), la seule matérialité pour la femme, pour la danse, pour la danseuse, comme pour tout le reste à l'exception de la nature, aura été celle des mots.

((Arôme de fraîchin par son geste dégagé de tout tissu, de tout rets. Comme si elle n'avait jamais été vêtue. Sa liberté est là, dans ce corps qui n'est pas le sien. Une peau enfilée par-dessus une peau enfilée par-dessus une peau. Preste et taciturne, elle transmigre sans heurts d'une peau à une autre, délaissant aussitôt les peaux chues de ses mues. Les enchantant aussitôt par une invocation muette qui les rend invisibles, car elle a lieu à côté. Capteur d'œil et d'efflorescence saline. Elle prend soin de se réserver les passages - les secrets de son art qui est l'art de l'entre-deux - et captive ailleurs le regard, ailleurs la brèche qu'ouvre son mouvement dans les corps et dans l'air. Pudeur interstitielle.))

Il y a une réponse matérielle à la divagation de Mallarmé. Il a écrit que son esprit se mouvait dans l'éternel. Nous écrivons que toute trace 
est un cycle. Qu'il n'y aura trace de la danse issue d'une blessure violente. Qu'il n'y aura trace de la danse dans la défécation parce qu'alors (c'est ce que dit Artaud) la matière vient d'ailleurs. Qu'il n'y aura trace de la danse qui soit le résultat d'un artifice, $d^{\prime}$ une adjonction faite au corps - à la viande, dit Deleuze devant une toile de Bacon. Qu'il ne peut donc y avoir trace de la danse que féminine, parce que le sang des menstrues prête sa noblesse à l'encre, quand, alentour, il fait bon y retrouver l'abjection de toute éternité. La danseuse écrit au sol avec son sang: modèle et idéal de trace dont rêve ma divagation.

((Parfois liant si bien les mouvements qu'on les croirait un, continu, infini, visible maintenant du début à la fin, elle nous lie à notre insu et nous voilà à sa merci alors qu'ébahis, nous rêvons qu'elle risque sa vie. Qu'elle risque sa peau. Elle fait davantage. Elle s'écorche vive, change de peau et recommence.))

En connivence avec la voix de Georges Rodenbach, il faisait de la danseuse dans sa " presque nudité », un " instrument direct d'idée » ("Les Fonds dans le Ballet »). Le poème aspirant à la pureté d'un mouvement condense chez lui les images, garrotte les bordages du vaisseau qu'il devient (oui, son « Salut » des Poésies: « Nous naviguons, ô mes divers / Amis, moi déjà sur la poupe »), se resserre phonétiquement, écope le sens, se délite tardivement quand « Rien, cette écume, vierge vers " arrive au seuil de sa réalité, à la fin du poème. Alors un rien ou un silence authentifié jaillit dans son évidence, celle de la page lue: « Le blanc souci de notre toile. » Voilà le chemin d'une pratique de lecture à laquelle Mallarmé a consacré autant d'énergie, peut-être plus, qu'à l'écriture elle-même. Clef de son axiome: notation d'une lecture $\mathrm{du}$ mouvement. Au titre d'une mathématique du rythme, ou d'une parenté du mouvement oculaire, on a pu prétendre que le « Coup de dés " réalise une chorégraphie du langage. Aucune trace (de la danse) ne provient du gradient ou de l'expression mécanique, musculaire, de l'excitation. La connaissance exactement pondérable transmise lors $d^{\prime}$ un rapport de communication est une imitation. Tout ce qui peut être lu sans imposer l'imitation est le témoin d'une fécondation détournée de son cours.

((Pourtant le rêve de l'arrêt persiste. L'entre-deux dérobé donne à voir - car invisible, on le voit plus clairement que si elle se mettait en corps de le représenter - donne à voir la statue. La vision à laquelle nous n'accédons jamais, dont nous rêvons chaque nuit, en sueur, remuant jusqu'à se découvrir dans la nuit tout en restant engouffré dans une immobilité profonde. Donne à voir SOI STATUE. Parce qu'elle bouge sans arrêts, de son geste émergent d'innombrables 
pétrifications, des statues de chair irriguée, sans peau, mais qui ne palpitent plus. Elle sème des arrêts. Aussitôt des statues se lèvent, aussitôt s'évanouissent. Du moins nous faut-il le rêver.

Car le danger des rets persiste. Elle danse tant qu'on s'oublie.))

Ce qui coule est un métal qu'on épure. Le sang a un goût de fer. Avant de désigner l'espace d'un théâtre qui borde la scène, traditionnellement, sur trois côtés, la coulisse est un décor mobile. Avant d'être le canal obscur et encombré où l'homme coiffé d'une perruque, d'une buse, d'un canotier projette le fantasme d'une étoile monnayable ou d'un rat apprivoisé, où il promène son désir de la rencontre d'un costume abondant et d'un costume nu (l'étoffe et le collant; le parapluie et la machine à coudre), la coulisse indique un mouvement et un objet indifférenciés. Elle est un paravent derrière lequel rien ne se passe. Une tentation consiste à regarder derrière, à faire de ce rien le dieu manquant. Le dieu du manque. C'est une seule et même fiction théâtrale, médicale, théologique et théorique qui interprète la coulisse comme l'indice d'une vérité où il faudrait pénétrer. Il ne s'agit pas de croire. Mais le rêve de mettre un doigt dans la plaie à défaut de voir la lunaison nous tiraille. C'est le regret.

((Mais vestale protectrice : pour chaque rêve un feu.))

Le Moyen Âge vouait un culte aux plaies du Christ et aux instruments de sa crucifixion. On retrouve des marques de cette adoration datant de la fin du XVII ${ }^{\mathrm{e}}$ siècle dans le monde germanique. La sensualité de ces images est étonnante. Nous ne pouvons manquer d'y voir les figurations de l'appareil reproducteur de la femme et de l'homme. Un clou a les extrémités arrondies et l'une est maculée de sang. Deux plaies ovoïdes, traversées par une mince ligne médiane, laissent échapper de petites formes sinueuses qui représentent des gouttelettes. Elles évoquent moins les spermatozoïdes que les menstrues. Ce sont les trois gravures qui ornent une feuille de prière publiée par J. P. Steudner à Augsbourg, aujourd'hui au Germanischer Nationalmuseum de Nuremberg. Celui ou celle qui priait lisait et disait s'incliner devant la blessure sacrée, implorer le Christ de guider ses pas vers la félicité éternelle, sur le chemin de sang. Les plaies douces comme le miel devaient assurer la rédemption des péchés lors du jugement dernier, seulement si le Christ les montrait à temps au Père. Que ces images n'aient pas eu la force d'évocation sensuelle qu'elles exercent sur nous n'infirme pas l'hypothèse de leur retour périodique. Les plaies détournent moins des péchés de l'humanité le regard du Père qu'elles ne cachent, aux yeux des fidèles, le rien qui préside au jugement. Croire en Dieu, c'est croire en la pureté. Croire en la pureté, 
c'est croire qu'il y a et a eu perte.

((Corps carcéral en mouvement perpétuel. Elle n'est autre que corps, corps en lui-même qui renverse toutes les expériences où il se couvre d'écailles. Ou de peau. Nulle place libre pour loger du regard quelque fleur, quelque épine élancée de futaies soudain hors des gangues de l'invisible, quelque chat que ce soit. L'empire qu'elle exerce vient de la chair et des os que rien ne soustrait à la chair et aux os. Irréductible sentence abattue et relevée par sa main. Main: main; pied: pied; clavicule: clavicule. Jamais déployés de la sorte cependant. Pris dans l'ubiquité verticale de deux points qui étalent la prison du mouvement. Prisonnière d'une nudité humaine effroyable. Mais voyageuse aussi, traversant tous les règnes sans soumission à un règne. Elle poursuit ses barreaux. Les treillis où grimper. L'ombre zébrée qu'elle sciera en posant amoureusement son corps dans la lumière.))

Alors l'axiome plus intime: Mallarmé écrit à Eugène Lefébure, dans une lettre datée du 27 mai 1867, la répulsion que lui inspirent les menstrues. C'est un fragment détaché qu'il joint à son envoi postal. Notation circonstancielle et, cette fois, le spectacle de la « bêtise de la Vie » est lu pour tirer leçon, parallèlement à une année éprouvante de crise physique et spirituelle, de cette expression: les « affaires ». L'homme et la femme qui se contentent d'incarner l'élément passif des " nécessités sociales" s'y rejoignent. Le mot devient la matrice de l'abjection. Ne versons pas trop vite le caractère acerbe des mots du poète au compte de la misogynie fin de siècle. La poésie observe avec suspicion le régime commercial et les bouleversements physiologiques parce qu'elle sait leur transposition dans le langage appauvrissant la relation symbolique. Affaires, les transactions infiniment reproduites, les fluctuations du numéraire qui signent un ordre symbolique dégénéré alors que, écrit Mallarmé dans le texte " Or ", " une liquéfaction de trésor rampe, rutile à l'horizon » lors des naturels et « fantasmagoriques couchers du soleil ». Il s'agit de ne pas enfermer le symbole dans le nombre, de ne pas classer le sang sous l'image médicale d'un transit d'humeur ou sous l'image mercantile d'un échange conventionnel. La sécrétion n'est pas une séparation. La sécrétion confond, comme la coulisse, le séparé et l'action qui l'amène à être. Seule une femme peut réellement écrire la danse. Le rythme animal périodique fond la trace et le mouvement. L'art peut dégager la trace et le mouvement de la génération. Mais il ignore le rythme qui lui donne corps.

((Si un éclat surnaturel l'auréole et la fait briller de feux claquants, 
d'éclairs trébuchants jusqu'à l'œil, si un éclat l'évacue de son corps et du monde des corps, je l'y mets. Le regard invente la lumière qu'il reçoit. Elle se meut ailleurs. Jamais d'ailleurs. L'autre monde qui la nimbe, elle l'ignore. Celui de tous, le mien, l'autre monde mien si je regarde bien. Mais combien d'exclusions faudra-t-il pour la voir simplement la voir là sans enluminer ses errances? Rassurant écart qu'affabule tout œil vain pour lutter contre sa propre danse banale.))

Mallarmé sacrifie au dieu du manque. Il écrit ce fragment détaché de la lettre à Lefébure :

Et l'un et l'autre s'affirment par ces misères, (qui seraient de grandeurs si elles étaient parvenues à leur Beauté, - quand la Femme, devenue au lieu de la Maladie la Destruction est courtisane, ou l'Homme, devenu au lieu d'un cerveau un Esprit - ) ils $s^{\prime}$ affirment, les superbes, dis-je, par ces misères, et répondent avec cet air de Mystère - qui n'a pu s'effacer même en ces tristesses, tant c'est la marque indélébile de Beauté - même de la Beauté de la Bêtise - " J'ai mes affaires. " Signifiant tous deux deux choses différentes d'aspects menteurs, mais identiques au fond.

Il maudit le crime inéluctable de la séparation.

((Elle ne brille ni n'encrasse sa posture quand tombe le rideau. Peu importe les feux, elle est même - d'une peau retrouvée pour une fois reconnue mais encore grossièrement - $d$ 'une peau qui lui sied le temps qu'il faudrait pour croire qu'elle en change une seule fois. Corps sans fard tout aussi lumineux. Corps de jour qui croupit comme ailleurs sous les feux.

Regardons mieux. Elle restitue dans son règne l'effroyable souveraineté d'y appartenir. Regardons-nous aussi.))

La seule atteinte peut se faire par les yeux. Ce que nous appelons le corps doit être identique $a u$ début à la fin. L'accessoire est une insulte au mouvement. Rien ne doit le gêner. Le sol doit être le dessin de ce corps même. La trace, un fragment du corps qui n'a pas été séparé. Le mouvement, un (re)jet de sang. Ce sont les conditions de la trace de la danse. Nous écrivons menstrues de préférence à menstruations. Qui évoquent de trop près les mesures de l'apparence. Le terme menstrues, par sa concision, pointe. L'instant où le rythme est visible. Le reste du temps oscille entre trop de sensations, variations climatiques, balancier d'attentes et de dénouements, pour octroyer une vue. Nous imaginons que le sang suit son cours parce que des spasmes nous sont audibles la nuit. Le rythme visible est une effraction du néant. 
((Lorsqu'elle sortit du halo terrestre venu - c'est un leurre - d'en haut, le moment fin d'un clignement d'œil, elle disparut moins qu'écartée ne l'est. Corps imprimé à l'air nocturne, visible sans phosphorescence ajoutée. D'abord cet ongle, puis cette phalange aux allures d'aile (si l'on se prête aux échanges anodins), ce poignet mat, l'aisselle en virgule pivotante ou l'espérance imagée du prochain être qui ne fuit pas au cœur des trombes sinueuses de ses jambes; puis tout le reste (qui pénétra peut-être avant l'ongle, permutant avec lui sa place de reste); son corps obscur ne semble plus et apparaît plus entêté. Elle réside au noir. La vue tricolore, son malheur, son accident perpétuel, sa damnation compatissante: fantasmes de nous.

Lorsqu'elle sortit du halo lumineux, rappelons-nous la joie solitaire qu'elle éventra au temps majeur d'une masse pressante.

Et nous plaignîmes, insensés, la tombée de cette NUIT PROVOQUÉE PAR LE GESTE.))

Son sang écrit la trace du mouvement. 